\title{
Specular surface reconstruction method for multi-camera corneal topographer arrangements
}

\author{
A. Soumelidis, Z. Fazekas, A. Bódis-Szomorú, F. Schipp, \\ B. Csákány and J. Németh \\ Computer and Automation Research Institute, \\ Budapest University of Technology and Economics, \\ Eötvös Loránd University \& \\ Semmelweis University, Hungary
}

\section{Introduction}

In a recent research and development project, a mathematically profound and technologically viable approach was developed to ensure a precise and reliable measurement and geometrical reconstruction of the human corneal surface. The intention was to get rid of the shortcomings of known corneal topographers and to produce reliable measurement results even for irregular corneas. With an eye on these expectations, a multi-view - i.e., a multicamera - measurement and reconstruction approach was proposed and used in the experiments. This approach and the lessons learnt from the implementation and testing of the topographer arrangement are presented in this chapter.
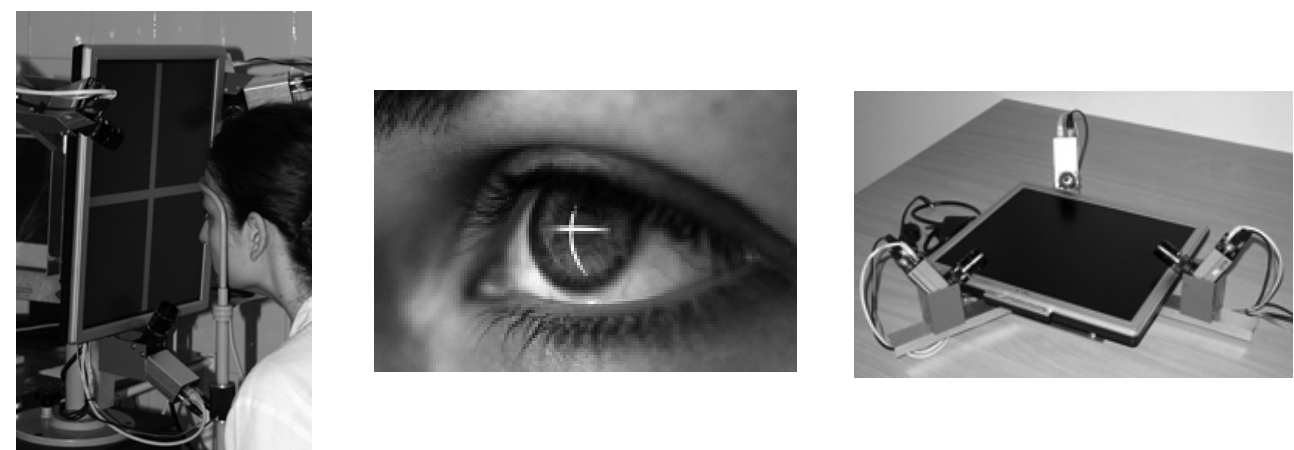

Fig. 1. The three-camera corneal topographer arrangement used in the measurements described in this paper (left). Virtual image of a bright cross-shaped test pattern (middle). Aside from the test pattern, reflections of the patient's eye-lashes and nose are also discernible in the image. The optical subsystem of the topographer arrangement (right).

Firstly, however, some background material on the measured organ (the human eye), and particularly, on its frontal section (the cornea), on its measurement methodology (corneal 
topography) and on the principle of the corneal measurement (specular surface reconstruction) are given. It is followed by a detailed presentation of the proposed measurement method. Finally, conclusions are drawn and future work is outlined.

\section{The human cornea and its geometrical measurement}

In technical terms, the human eye can be considered an imaging sensor with its frontal section responsible for focusing the incoming light rays and its rear section responsible for converting the image - formed on its internal surface - into electrical signals available for further processing. The cornea - located in its frontal section - is the primary optical structure of the human eye. The corneal tissue is transparent. The human cornea is an optical structure, which generates about the $70 \%$ of the total refractive power of the eye, while other structures in the light-path - including also the crystalline lens - contribute significantly less the total refractive power.

The outer corneal surface itself is not an optical surface. It is the coating of the corneal surface, i.e., the pre-corneal tear film that provides a smooth optical surface. The tear film is replenished with every blink and after its build-up; it provides a smooth optical surface over the microscopically irregular corneal surface (Németh et al., 2002). The frontal and the inner surface of the human cornea together contribute about 43 dioptres to the eye's total refractive power, which is about 65 dioptres. Physiologically, the shape of the frontal corneal surface is close to spherical.

Primarily, it is because of the cornea's mentioned high share in the total refractive power that the detailed description of the corneal surface and of its refractive power map is of considerable value ophthalmic diagnosis. For this reason, it is not surprising that the corneal measurement devices, such as keratometers, corneal topographers, and methods - aiming at measuring and determining the corneal shape and using it for further optical calculations - have a relatively long history; bibliographical details of some milestone papers written by Helmholtz, Purkinje, Placido and Gullstrand are given in (Jongsma et al., 1999).

Purkinje images are virtual images reflected from the consecutive surfaces of the light-path, namely from those of the cornea and crystalline lens. These reflections are known since the first half of the nineteenth century. There are four Purkinje images corresponding to the anterior (1st Purkinje image) and posterior (2nd) surfaces of the cornea, the anterior (3rd) and posterior (4th) surfaces of the crystalline lens. The first Purkinje image is the brightest. As the size and shape of this image depends on the geometry of the frontal corneal surface, it can be used for measurement and surface reconstruction purposes. Most of the keratometers and corneal topographers use this principle. Another important application field of the Purkinje images are the eye trackers.

Presently, corneal topographers are used in a range of examinations in ophthalmic diagnosis, see e.g., (Corbett et al., 1999) for a good overview of the topic. A selection of corneal topographers is reviewed in (Jongsma, 1999). The majority of these measurement devices and methods rely on the specularity of the cornea, or more precisely, that of the pre-corneal tear-film and use the 1st Purkinje images. These methods are referred as reflection-based methods, and the topographers using these methods as reflection-based topographers. In case of such topographers, a bright measurement-pattern of known and well-defined geometry - e.g., concentric rings called in this context Placido-disk, see Figure 5 - is generated and displayed in front of the patient's cornea. The virtual image of this pattern is photo- 
graphed by one or more camera. The distorted virtual image, or images are then analyzed, and the corneal surface is mathematically reconstructed. Based on this reconstruction, height- and refractive power-maps are produced and displayed for inspection by the ophthalmologist.

Cornea topography is an important field of ophthalmologic diagnosis. The purpose of a cornea topographic examination is to determine and display the shape and the refractive power map - that is, the (local) measure of the focusing effect caused by an optical structure (e.g., a lens, or a refractive surface) - of the living human cornea. This contributes to the total refractive power of the eye that determines whether the light-rays entering the eye are properly focused on the fovea, or not. The refractive power of the cornea varies slightly over the corneal surface. The cornea topographers usually display these location-dependent refractive power values - i.e., the corneal refractive power map - as pseudo-coloured maps, see Figure 3. From this map, optical aberration features are calculated for the measured cornea by the topographers, or by some standalone programs. After a corneal measurement, the ophthalmologist visually inspects the corneal map, considers the optical aberrations mentioned above, other available relevant measurement data (e.g., the optical aberrations of the whole eye computed from measurement data produced by a Shack-Hartmann wavefrontsensor) and circumstances. Then she suggests an appropriate treatment - if necessary - for the patient's eye. Corneal topography plays a significant role in the diagnosis of corneal diseases, in contact lens selection and fitting, in planning sight-correcting refractive surgical operations, and in their post-operative check-ups.

In case of healthy and regular corneal surfaces, the presently available corneal topographers generally produce good quality corneal snapshots, and based on these, precise and reliable maps are generated. However, even for healthy regular surfaces, small impurities and tiny discontinuities in the pre-corneal tear-film may produce extensive measurement errors. This is because the simplistic measurement patterns, such as the aforementioned Placido-disk that are still widely used in corneal topographers, do not provide the necessary information to correct such seemingly local problems. The formation of such a measurement anomaly can be followed in Figure 6. The resulting refractive power map exhibits a serious artifact. It arises as a result of the poorly tracked Placido-rings that give rise to some highly curved arcs, and these arcs in turn result in significant and extensive - i.e., non-local - errors in the generated optical power map.

The measurement anomalies could be even greater, if callused irregular corneal surfaces are examined using a Placido-based topographer. In such cases, many of the reflected ringpoints may disappear and it may be difficult, if at all possible, to determine the exact radial order of the ring-arcs. Consequently, only a partial, rough and unsatisfactory surface reconstruction can be achieved that is of no use for any diagnostic purposes. To this end several more informative measurement patterns were suggested, e.g., (Vos et al., 1997). Some of the proposed patterns facilitate the identification of point-correspondences by using positioncoding colour patterns, e.g., (Griffin et al., 1992; Soumelidis et al., 2008).

Another source of measurement anomalies is the dynamic behaviour of the pre-corneal tear film. It should be taken into consideration even when carrying out a single (i.e., nondynamic) corneal topographic measurement. The pre-corneal tear film coating the corneal surface keeps changing between blinks. In the first few seconds after a blink, the tear film spreads over and builds up on the corneal surface. Then, it begins to evaporate and after a while it gradually breaks up. The optical smoothness of the corneal surface is guaranteed 
only during the evaporation phase of the pre-corneal tear film, not during its break up. Snapshots taken at other times exhibit various degrees of measurement anomaly. Furthermore, weird and incorrect optical power maps are produced, if the measurement pattern has not been properly centred onto the cornea by the ophthalmologist, or if the image taken was blurred because of the improper camera-eye distance setting used.

There are some alternative image-based examination methods of the cornea. For example, the slit-scanning method is based on the physiologic Tyndall phenomenon of the corneal stroma. Using a narrow slit-like light beam, images can be obtained from a line of the surface. This method may be combined with the reflective one. Another example is the Scheimpflug imaging, which is a photographical technique. Both the front and rear surfaces of the cornea can be examined with this method. Optical coherence tomography examination of the anterior segment is based on low coherence interferometry (Swartz et al., 2007). All the mentioned alternative imaging methods are line-based, i.e., they process one scanline in a moment. This way of operation, however, constitutes a major drawback when compared with the reflective imaging method.
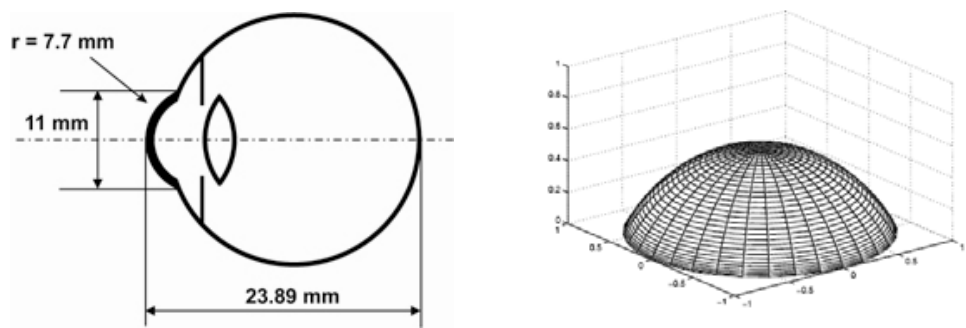

Fig. 2. In Gullstrand's eye model (left), the corneal surface is represented by a spherical calotte surface (right).

\section{Eye models, cornea models}

The Gullstrand eye model - since its creation in 1911 - had been the basis of optical modelling and calculations concerning the human eye for a long period of time. In this early eye model, like in many others proposed later, the refractive power of the cornea is represented solely by its anterior surface. This approximation describes the optical behaviour of living corneas - except for those having certain special ophthalmic conditions - reasonably accurately. This is because, in case of the typical corneas, one can estimate the whole corneal refractive power fairly precisely from the radii of curvature of the anterior surface.

Recent eye models - such as those proposed by Schwiegerling in 1995 and by Liou and Brennan in 1997 - are more realistic and more precise models with more tuneable parameters involved. One of the reasons for using more realistic eye models is to estimate the retinal image quality achieved. The latter model, for example, incorporates also the continuous change of the refractive index within the crystalline lens. The inclusion of such fine details into the model, however, does not prejudice the optical importance of the anterior surface (de Almeida \& Carvalho, 2007). 

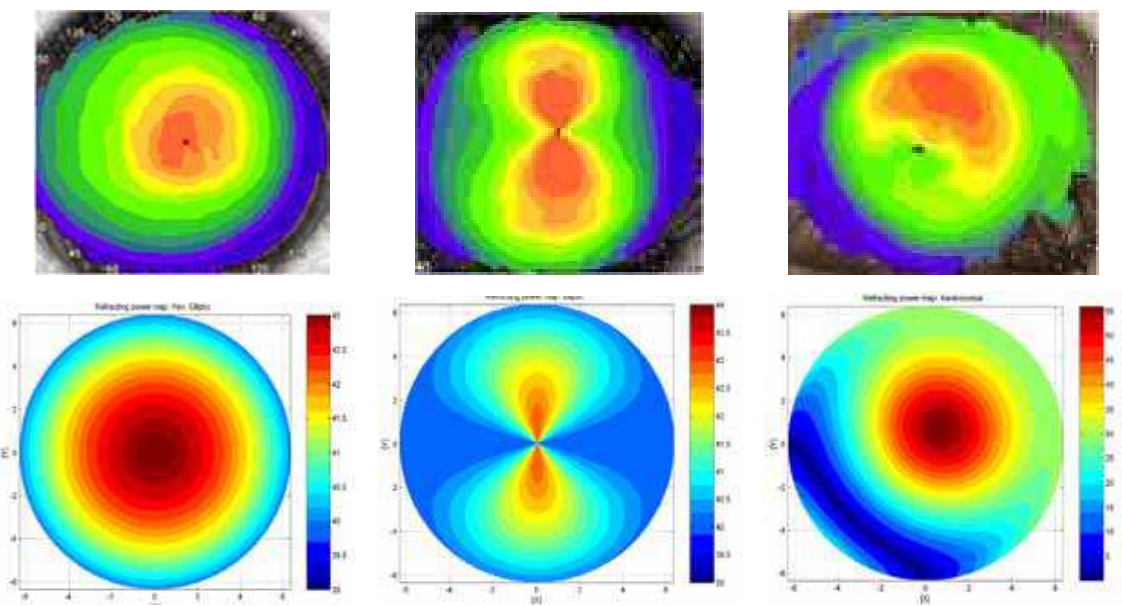

Fig. 3. Refractive power maps of healthy and abnormal human corneas (top row) and their corresponding test models (bottom row). Regions marked with red have the highest dioptre values in the above maps, while the dark blue regions have the lowest.

A test cornea is an optical device that serves as a physical model - a proxy - of the (human) cornea. It is primarily used for testing and calibration purposes in conjunction with corneal topographers and other corneal measurement devices. If used with reflective corneal topographers, the test cornea must have a reflective optically smooth surface. The shape and the size of the test cornea should match those of the "average" human cornea. A test cornea is shown in the left image of Figure 4.
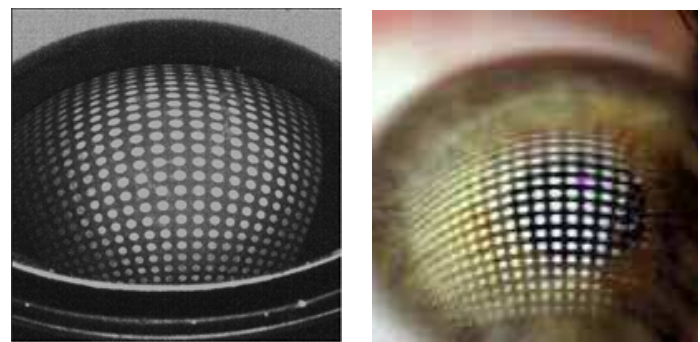

Fig. 4. A virtual image produced by a spherical test cornea (left) and that produced by a living cornea (right).

A test cornea looks like plano-convex lens; except for it is not transparent. Usually, it is fixed onto a planar holder with its planar side. The elaborate part of the test cornea is its convex side; it must be a high precision optical surface. The precision of the planar side is less important, though it still plays a significant role in the positioning of the test cornea. Test corneas are usually made of some opaque machinable material (e.g., dark glass, ceramics, metals, plastic) appropriate for producing high-quality optical surfaces.

In case of glass and ceramics test corneas, these can be manufactured using the so-called single-point diamond turning process. In case of metals (e.g., stainless steel), a customary high-precision lathe can be used for turning. Among the plastic materials used in optics, the 
polymethyl-methacrylate (PMMA) is probably the best candidate material for a test cornea. (As in case of the glass used for this purpose, also the PMMA material has to be dark.) There is considerable manufacturing experience with respect to PMMA, as spectacles, and contact lenses are known to be manufactured from this plastic.

Test corneas that are most commonly used in conjunction with corneal topographic measurements have spherical calotte surface. Such a test cornea is shown in Figure 4 together with a living cornea photographed from a similar angle for comparison. The radius of such a test cornea is chosen to be equal to the radius of curvature of the typical human cornea. However, different eye models specify and use slightly different "typical" dimensions. It should be noted here that the surface of a living human cornea is not a perfectly spherical surface. This fact is taken into consideration by a number of eye models; as these regard the "typical" corneal surface as ellipsoid surface. Even if the spherical test corneas are considered a simplistic physical model of the cornea, they serve as the most common calibration devices for corneal measurement instruments in respect of general calibration. Clearly, such test corneas are of no use for more complex and more realistic sensitivity analysis purposes, such as sensitivity of the measurement regards to optical aberrations, see e.g., (Wang, 2006); furthermore, they do not facilitate testing of surface reconstruction algorithms.

Three test surfaces were considered essential for testing purposes in the mentioned project. Note that further corneal test surfaces are specified in (ANSI, 2008). Two of these three surfaces are surfaces of revolution; therefore, they can be manufactured by precision turning. However, the third one is a free-form surface which cannot be manufactured by turning.

Surface modelling a healthy cornea is a slightly more realistic model than a spherical calotte model. The surface is a surface of revolution, characterized by two radii of curvature; one for the central region, i.e., near the axis of revolution measured in an axial plane, and one for the periphery, i.e., near the brim of the test surface also measured in an axial plane. Between these two extremes, the local radius of curvature should change in a linear fashion, or - as it would results in a function that cannot be given explicitly - according to some smooth continuous function. In practice, the test surface can be chosen to be the calotte of a prolate spheroid. The refractive power map of such a test surface is shown on the left-handside of Figure 3. In this case, the central refractive power was set to 43 dioptres, while the peripheral refractive power was set to 40 dioptres. These are typical values for healthy subjects.

Regular astigmatic corneal surface is a typical degenerative deformation of corneal surface. Plainly speaking, the corneal surface becomes somewhat cylindrical. It is characterised by the direction of the magnitude of the astigmatism. In case of an astigmatic corneal surface, the local curvature and consequently the local refracting power vary along and perpendicular to - and for that matter, skew to - the direction of astigmatism differently. A simple astigmatic surface is the calotte of a prolate spheroid (cut from a prolate spheroid by a plane parallel to the axis of revolution). The refractive power map of such a test surface is shown in the middle image in lower row of the Figure 3. There, the direction of the astigmatism is chosen to be horizontal, furthermore, the horizontal and vertical central refractive powers were set to 42 dioptres and 45 dioptres, respectively; while the peripheral refractive power was set to 39 dioptres, respectively.

Keratoconic surface is a characteristic degenerative deformation of corneal surface. It is characterised by a relatively steep conic upthrust in the corneal surface. For modelling puposes, the base surface can be chosen from the aforementioned test surfaces, while the kera- 
toconic upthrust can be modelled as the base surface being multiplied with a unimodal function. This will result in a free-form surface that cannot be manufactured by turning, a but milling. The refractive power map of a keratoconic test surface is shown on the righthand-side of in lower row of the Figure 3.

\section{Specular surface reconstruction approaches}

The mathematical reconstruction of a smooth specular surface from the distortions it brings about to some known, well-defined measurement pattern, called fiducial, when this measurement pattern is viewed and photographed in the virtual images, is an active research area. The reconstruction can be local or global. In case of local reconstruction, sufficient conditions are given for the uniqueness of the reconstruction in (Savarese \& Perona, 2001). The formulae to determine the actual surface-patch are given in (Savarese \& Perona, 2002a). Multi-view methods for the practically more important global reconstruction were published recently. These methods rely on the smoothness of the surface and use several views to make the unique reconstruction possible. For an unknown smooth, convex specular surface - viewed by several cameras - those points are located on - or near to - the specular surface for which the unit normal vectors - calculated from the reflections at these points are approximately the same for different views. This observation is the basis of the voxelcarving method suggested by (Bonfort \& Sturm, 2003). Clearly, this method has an important limitation; it can be used only for those surface portions that reflect the measurementpattern into more than one camera. An elegant surface reconstruction method was proposed by (Kickingereder \& Donner, 2004). There the light-reflection at the surface is described with a total differential equation. The mathematical reconstruction of the surface is achieved by the numerical solution of this total differential equation.
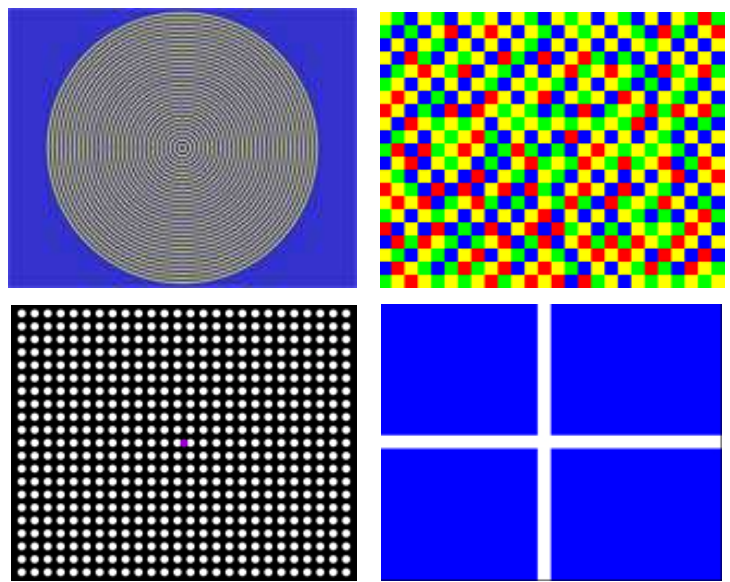

Fig. 5. Various measurement patterns used in our experiments and measurements. Placido disk consisting of concentric rings (top left). A position-coding colour checkerboard (top right). A square grid of circular spots (bottom left) with its centre marked. A cross-shaped pattern (bottom right) used only for basic checks. 
The purpose of the measurement patterns is to facilitate the identification of the mathematical mappings - produced by the specular corneal surfaces - by providing spatial correspondences between the elements of the measurement pattern (e.g., circles, characteristic points) on one hand and their images on the other. A great variety of measurement patterns is used in the reflection-based corneal topographers. Here, we consider planar patterns only; though, measurement patterns do appear in various topographers on spherical, paraboloid of revolution, and other surfaces.

In Figure 5, a selection of planar measurement patterns are shown. These were used either for measurement, or test purposes in the experiments reported herein. In the top left image, Placido's disk is shown. It consists of bright concentric rings. This measurement pattern supports the circle to curve correspondence, but does not support a point-to-point correspondence; and as explained in (Sicam, 2007), for this reason the Placido-based corneal topography gives rise to skew ray ambiguity and as a consequence theoretically inadequate for surface reconstruction. Though, to be fair with the Placido-based topographers, the effect of skew ray ambiguity is insignificant in most of the corneal surfaces.

A position-coding colour checkerboard is shown in the top right image of Figure 5. A somewhat similar colour-coding approach was proposed earlier for another application field in (Griffin, 1992). The generation of the position-coding colour checkerboard shown in the figure is outlined in (Soumelidis et al., 2008). A square grid of circular spots with its centre marked with a coloured spot is shown in the bottom left image of the figure; while a crossshaped pattern - used only for basic settings and checks - is show in the bottom right image of the same figure.
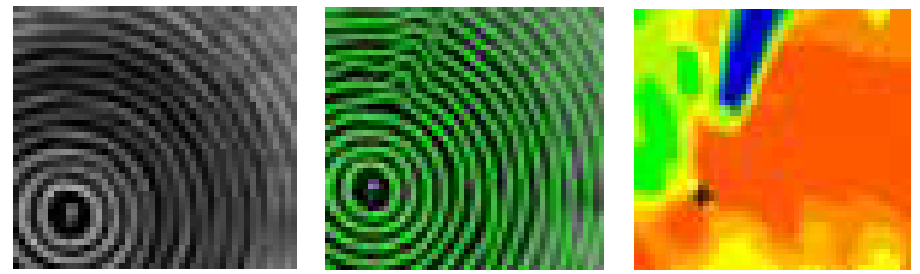

Fig. 6. A measurement artifact (i.e., the blue wedge in the right image) arising after a poor ring-tracking step (middle). The distorted rings of the Placido-disk (left). These rings do not facilitate their local identification.

\section{An experimental multi-view corneal topographer arrangement}

\subsection{Arrangement with up to four cameras}

The surface reconstruction method proposed in (Fazekas et al., 2008) - and described herein in more detail - was originally developed for the experimental corneal topographer arrangement described in (Soumelidis et al., 2008). In the following, this arrangement will be referred as the target-arrangement. As it will be apparent later on, the proposed method is not limited to the target-arrangement; however, up to now, it was tested only on that testbed. The camera-system of the target-arrangement may comprise at most of four cameras due to some internal networking limitation. Presently, however, a three-camera topographer arrangement is used for the experiments. The camera-system is mounted rigidly on a high-quality TFT-display which serves as measurement-pattern generator of the topographer arrangement. The target-arrangement is shown in Figure 1, and schematically in 
Figure 9. Clearly, this arrangement is very delicate and needs proper calibration for any meaningful measurements. The individual and joint calibration steps of its cameras are outlined below.
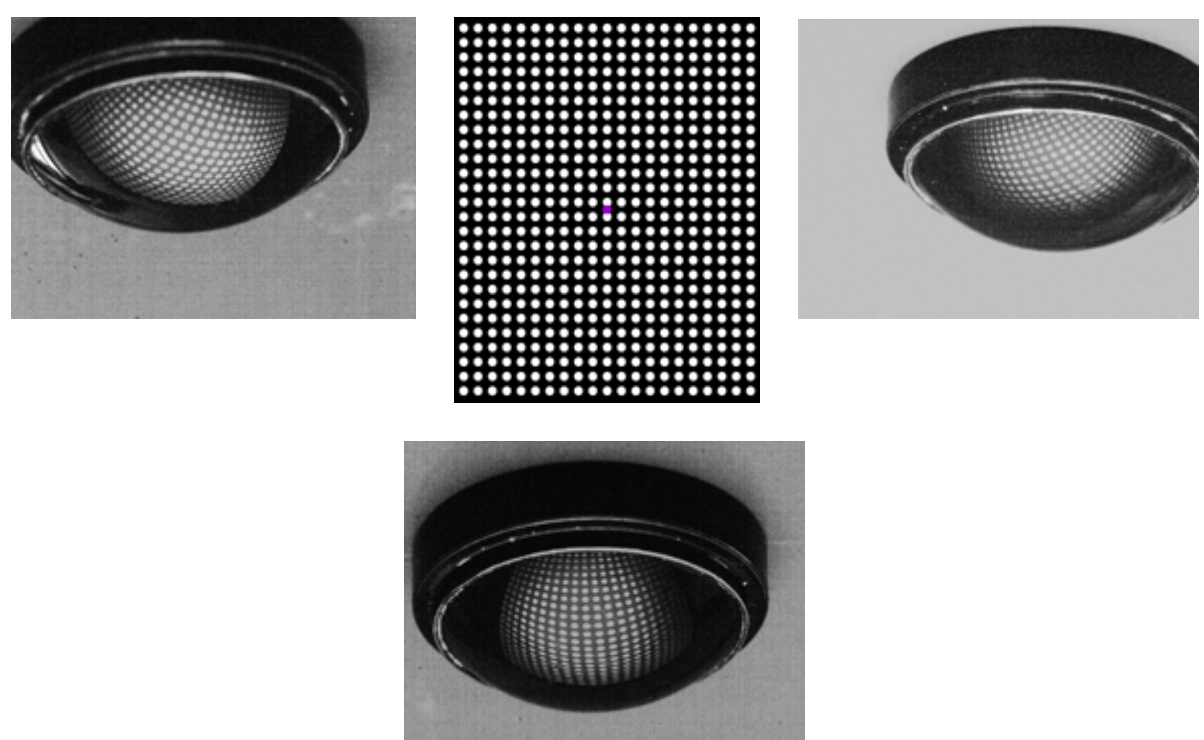

Fig. 7. Distorted virtual images (left, right, bottom) of the measurement pattern (centre) reflected by the specular spherical surface of a test cornea - as "seen" by the three cameras of the topographer arrangement.

\subsection{Individual calibration of the cameras in the arrangement}

In the recent years, camera calibration and position estimation methods based on planar fiducials have gained popularity in the stereo vision community in respect of spatial reconstruction problems. One of the reasons behind this popularity is the fact that these fiducials are easy to produce; they can be printed on a desktop laser printer in acceptable quality; therefore their production is fairly inexpensive.

A general camera calibration algorithm based on such fiducials is given in (Hartley \& Zissermann, 2004), while a concrete practical implementation - based on images of a checkerboard placed in different orientations and positions, and photographed with the camera to be calibrated - was proposed in (Zhang, 2000).

Firstly, the calibration of a single camera is outlined below. For a more detailed description of this algorithm - used in a different context - see (Bódis-Szomorú et al., 2008); the notation used herein without a detailed explanation is similar to that of appearing in the cited paper. The position of a checkerboard in the 3D space has 6 degree-of-freedom. With $m$ images taken, one has to determine $(9+6 m)$ camera parameters in total.

- Localisation of characteristic points in the image (i.e. the localisation of the image points corresponding to the corners of the black and white squares of the checkerboard) using an appropriate corner detector. 
- Estimation of the homography $\boldsymbol{H}=\left[\begin{array}{lll}h_{1} & h_{2} & h_{3}\end{array}\right]$ - for each checkerboard position photographed - existing between the characteristic points of the planar checkerboard and their corresponding image points.

- Estimation of the intrinsic camera parameters based on the homographies. These parameters can be derived from the condition that image-points of the plane's complex-valued circular points lie on the image of the absolute conic $\omega$. See details in (Hartley \& Zissermann, 2004). $\omega$ can be estimated with a least-square method from the homographies from conditions $\boldsymbol{h}_{1}^{T} \omega \boldsymbol{h}_{2}=0$ and $\boldsymbol{h}_{1}^{T} \omega \boldsymbol{h}_{1}=\boldsymbol{h}_{2}^{T} \omega \boldsymbol{h}_{2}$. Since $\omega=\boldsymbol{K}^{-T} \boldsymbol{K}^{-1}$, therefore the camera calibration matrix $K$ can be computed via Cholesky-decomposition. See details in (Zhang, 2000).
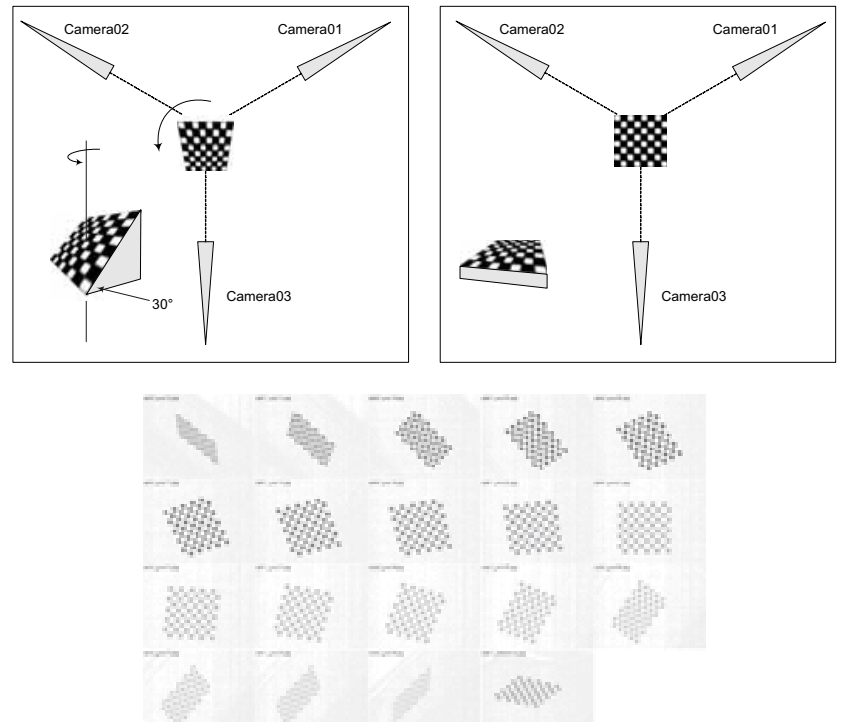

Fig. 8. A $10 \mathrm{~mm}$ by $10 \mathrm{~mm}$ checkerboard was used for the calibration of the cameras in the topographer arrangement. Firstly, the checkerboard was placed in a horizontal position and photographed with the cameras (top right). Then the checkerboard was fixed to wedge, turned around and photographed in a number of positions with the cameras (top left). The checkerboard images taken with one of the cameras (bottom).

- Computing the extrinsic camera parameters - for each image - based on $K$ and the appropriate $\boldsymbol{H}$ from $\lambda\left[\begin{array}{lll}\boldsymbol{h}_{1} & \boldsymbol{h}_{2} & \boldsymbol{h}_{3}\end{array}\right]=\boldsymbol{K}\left[\begin{array}{lll}\boldsymbol{r}_{1} & \boldsymbol{r}_{2} & \boldsymbol{t}_{W}\end{array}\right]$ condition.

- At this stage, estimates are available for all the parameters of the following linear camera model $\lambda \tilde{\boldsymbol{C}}=\boldsymbol{P} \tilde{\boldsymbol{W}}=\boldsymbol{K}\left[\begin{array}{ll}\boldsymbol{R} & -\boldsymbol{R} \boldsymbol{t}\end{array}\right] \tilde{\boldsymbol{W}}=\boldsymbol{K}\left[\begin{array}{ll}\boldsymbol{R} & \boldsymbol{t}_{W}\end{array}\right] \tilde{\boldsymbol{W}}$ for each of the images.

- Parameter optimisation using the Levenberg-Marquardt method with the following cost function to be minimised 


$$
f\left(\boldsymbol{p}_{\text {in }}, \boldsymbol{p}_{\text {ex }}^{(1)}, \boldsymbol{p}_{\text {ex }}^{(2)}, \ldots, \boldsymbol{p}_{\text {ex }}^{(m)}\right)=\sum_{i=1}^{m} \sum_{j=1}^{n}\left\|I_{i j}-\varphi\left(\boldsymbol{p}_{\text {in }}, \boldsymbol{p}_{\text {ex }}^{i}, \boldsymbol{W}_{i j}\right)\right\|_{2}^{2}=\sum_{i=1}^{m} \sum_{j=1}^{n} d^{2}\left(I_{i j}, \breve{I}_{i j}\right) .
$$

In order to calibrate a multi-camera arrangement based on the camera calibration method outlined above, one needs choose a reference checkerboard position and orientation in such a way that the checkerboard placed in that position and orientation can be properly "seen" by the cameras; furthermore, all the images taken by the cameras of the checkerboard in this position and orientation are appropriate for feature extraction. Among the series of checkerboard images shown in Figure 8, the checkerboard position appearing in the rightmost sub-image in the bottom row was this common reference position.

If, for instance, the camera parameter vector for Camera01 - derived via the above calibration process - is in the following form

$$
\boldsymbol{p}_{\text {Camera01 }}^{\text {all }}=\left(\boldsymbol{p}_{\text {in }}^{T}, \boldsymbol{p}_{\text {ex }}^{(1) T}, \boldsymbol{p}_{\text {ex }}^{(2) T}, \ldots, \boldsymbol{p}_{\text {ex }}^{(m) T}\right)^{T},
$$

and the $k$-th checkerboard-image is the one that was taken of the checkerboard in its reference position, then the parameter vector characterising the position and orientation of Camera01 with respect to the reference checkerboard-position is as follows.

$$
\boldsymbol{p}_{\text {Camera } 01}=\left(\boldsymbol{p}_{\text {in }}^{T}, \boldsymbol{p}_{\text {ex }}^{(k) T}\right)^{T} \text {. }
$$

The parameter vector for the other cameras, i.e., for Camera02 and Camera03, can be derived similarly. As shown in Figure 8, a checkerboard - with a size comparable to that of a typical human cornea - was used for the calibration of the cameras of the topographer arrangement. Firstly, this small checkerboard was placed in a horizontal position and photographed with each of the cameras.

Then the checkerboard was fixed onto wedge and was turned around and photographed in a number of positions with each of the cameras. Then with the method outlined above the camera parameter vectors were derived. The resulting geometrical positions and orientations of the cameras (of the calibrated three-camera arrangement) are shown in graphic reconstruction of Figure 9. The reconstructed arrangement is qualitatively acceptable.
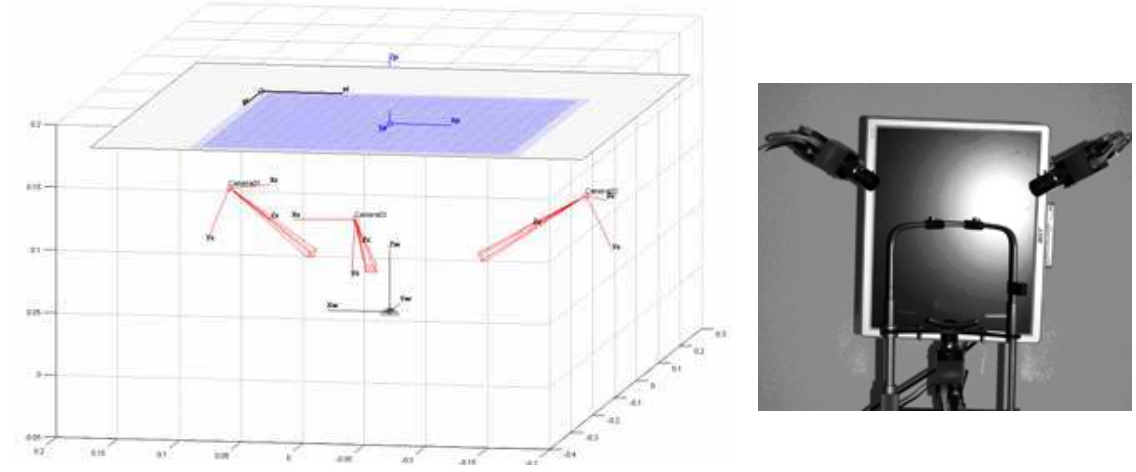

Fig. 9. The graphical representation of the calibration results (left). The pyramids represent the cameras. The three-camera arrangement - with the head-rest - for reference (right). 


\section{Determining the discrete and continuous distortions}

\subsection{Segmentation, feature extraction, filtering}

In our experiments, a rectangular array of bright circular spots - shown in Figure 7 - was used as measurement-pattern. The blobs in the reflected images - corresponding to the circular spots in the rectangular array of the measurement-pattern - were detected at a number of thresholds, and the centre-point and a few shape descriptors were calculated for each blob. The detected blobs were then checked for their areas. Blobs within blobhierarchies, - i.e., blobs that were more or less intactly preserved at a number of thresholds, see examples in Figure 10 top inset - and blobs that greatly overlapped with ones gained at other thresholds were merged with each other; as these were assumed to be the images of the same circular spots. Blobs with much bigger areas than those of the blobs in their neighbourhood were removed from the list of acceptable blobs, as these were considered be segmentation artifacts, rather than reliable segmentation results.

A distorted virtual image - formed by a test cornea - is shown in Figure 10. The process of segmentation, feature extraction, blob merging and filtering outlined above can be followed through images and insets of the figure. The area of each detected spot is indicated by that of a coloured disk.
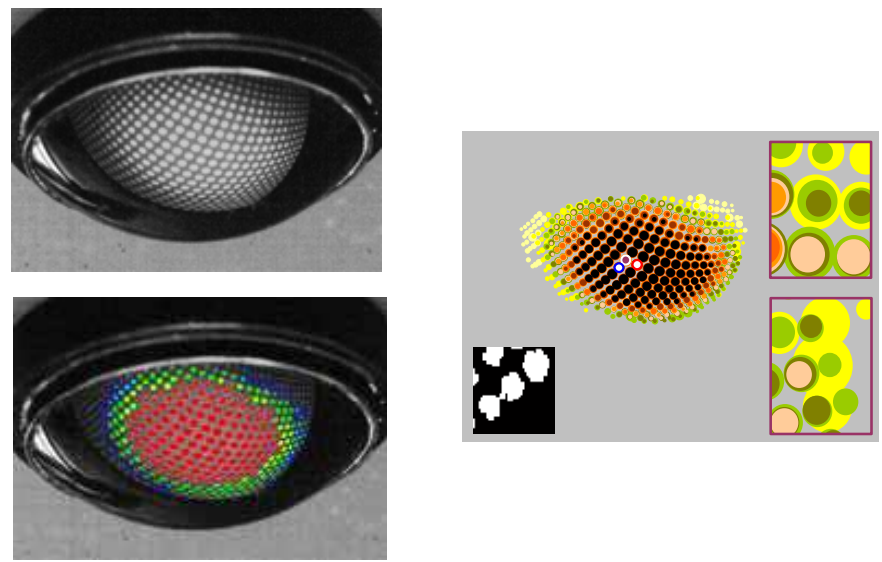

Fig. 10. The virtual image of a square grid of circular spots as being reflected by a test cornea (top). The areas of the spots detected at different intensity thresholds are indicated by that of the coloured disks (right). Blob hierarchies (see main text for details). (top inset). Blobs shown as yellow disks - split into two separate blobs each - shown as light green disks - at higher thresholds (right inset). A compound white blob - corresponding to two separate circular spots of the measurement pattern - appearing in one of the thresholded images (left inset). Consolidated - i.e., filtered - blobs (left) with colours indicating the highest threshold that produced a shape-wise and size-wise acceptable blob at that location.

\subsection{Tracking blobs within curved rows and columns}

A coloured circular spot, shown in the central image of Figure 7, was used for marking the geometric centre of the rectangular array. Blobs corresponding to these spots in the three reflection images were identified, and their centres were used as starting points for the sub- 
sequent blob-identification process. Each of the three 2D-point-sets - formed by the blobcentres of the blobs detected in the reflection images - was submitted to Delaunnay-triangulation, see Figure 11. A similar approach appears in (Trichet \& Merialdo, 2007) in the context of a different application.

The sides of the resulting triangles were then tracked - starting from the mentioned starting points - in "row" and "column" directions. As an example, the images of a living cornea taken by the three-camera topographer arrangement are shown in Figure 16. The results of the tracking process are shown in Figure 17.

For the 2D point-set shown in right-hand-side image of Figure 10, these directions are identified by the white ring to blue ring and white ring to red ring directions, respectively. Based on the tracking results, correspondences between the original circular spots and the detected blobs were established; in other words, a mapping from the original rectangular grid to each of the three point-sets was established.

For the purpose of the tracking, the side-lengths of the Delaunnay-triangles, the directions of sides and the area-ratio of the blobs - connected by the side concerned - were used.
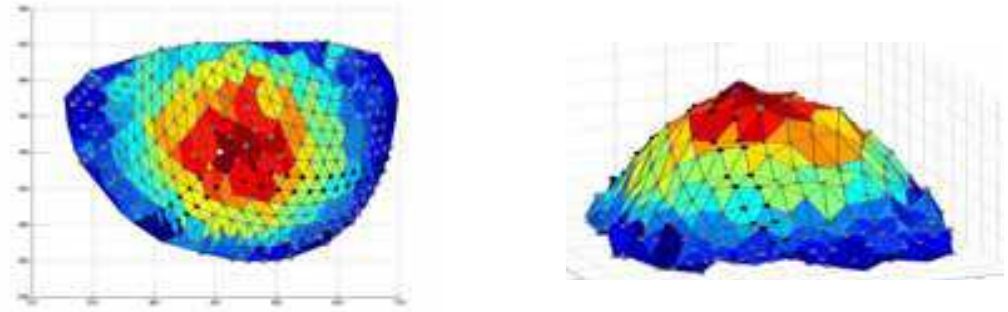

Fig. 11. Delaunnay-triangulation of the detected blobs' centre-points (left). The area of the corresponding blobs indicated in the z-coordinates and by the colour of the triangles.

\subsection{Approximation of the apparent distortions using splines}

Spline interpolation was used to convert the three discrete mappings to continuous mappings, thereby approximating the mappings (optical distortions, in this case) between the points of the measurement-pattern and those of the reflection images. A spline-based approximation of a known mapping is shown as an example in Figure 12.

Each mapping is approximated using two collections of splines, one parameterised with the "row" variable $\left(x_{1}\right)$, and the other one parameterised with the "column" variable $\left(x_{2}\right)$ of the measurement pattern's plane. With these two splines, the location of any intermediate image point can be calculated in two steps by creating interpolated image points first in "row" direction, and then fitting a spline onto these in "column" direction.

Having approximated the three continuous planar mappings, that is, one for each camera, now, we can turn our attention - once again - to spatial issues, namely, the surface reconstruction problem. 

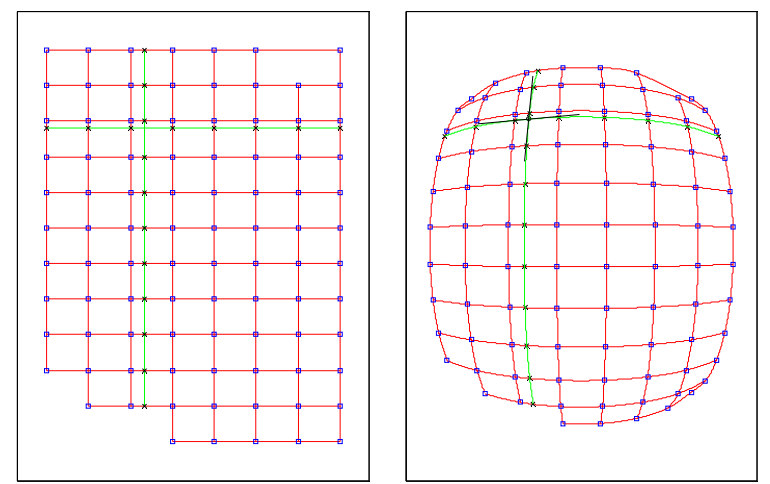

Fig. 12. A rectangular grid with some missing grid-points (left). The distorted image of the grid. A two interpolation lines and their corresponding interpolation curves are shown in green.

\section{Surface reconstruction from the continuous approximation of distortions}

\subsection{Mathematical model of light-reflection}

Mathematically, the smooth specular convex surface $F$ is described and sought in preferably chosen spatial coordinate systems. Each of these coordinate systems corresponds to one of the cameras of the topographer arrangement. For notational simplicity, only one of these coordinate systems is considered in the following formulae. The origin of the camera coordinate system $B$ is placed in the optical centre of the particular camera and the $z$-axis of this coordinate system is the optical axis of the camera. The specular surface $F$, i.e., the surface of an artificial or a living cornea is described in the following form:

$$
F\left(x_{1}, x_{2}\right)=S\left(x_{1}, x_{2}\right) \hat{x} \quad\left(\hat{x}=\left(x_{1}, x_{2}, 1\right)^{T}\right)
$$

Here, $S(x)\left(x=\left(x_{1}, x_{2}\right)\right)$ is a scalar-factor describing the inverse distance ratio, measured from $B$, of the 3D point $P_{x}$ - corresponding to $\hat{x}$ - and the surface-point appearing in the same direction as $P_{x}$ from $B$. The propagation of light from the points of the measurement pattern to those of the distorted image, i.e., $P_{y} P P_{x,}$, is described in the coordinate system.

By doing so, a mapping is identified between the points $P_{y}$ of the measurement pattern and the points $P_{x}$ of the image. This mapping is $P_{y} \rightarrow P_{x}$.

It follows from the conditions prescribed for the mathematical surface that mapping $P_{y} \rightarrow P_{x}$ is one-to-one. It follows from the physical law of light reflection that the two-variable function $S(x)$ describing surface $F$ satisfies the following first-order partial differential equation (PDE). 


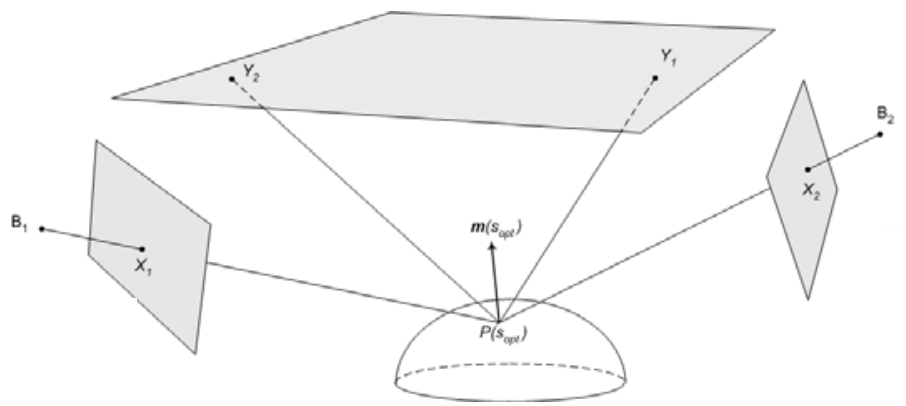

Fig. 13. Two points $\left(Y_{1}\right.$ and $\left.Y_{2}\right)$ being reflected by the same patch of the specular surface into the two cameras; $m$ is the normal of the surface patch.

$$
\frac{1}{S(x)} \frac{\partial S(x)}{\partial x_{j}}=\frac{v_{j}(x)-x_{j}}{\left\langle\hat{x}, \hat{x}-v_{j}(x)\right\rangle} \quad(j=1,2), \quad v(x)=|\hat{x}| \frac{k+f(x)-S(x) \hat{x}}{|k+f(x)-S(x) \hat{x}|} .
$$

Function $f(x)$ can be expressed with mapping $P_{x} \rightarrow P_{y}$. Here, $k$ is a vector pointing to a reference point in the plane of the measurement pattern; while $\langle. .$.$\rangle denotes the scalar product of$ the 3D space. It follows from the mathematical model described above that surface $F$ can be determined uniquely under the starting condition of $S(0,0)=s_{0}$, if the $P_{y} \rightarrow P_{x}$ mapping is known.

In Figure 13, the same surface-patch - with unit normal $m$ - of smooth specular surface $F$ reflects into the two cameras according to the physical law of light reflection.

\subsection{Joint camera calibrations using a test-cornea of known dimensions}

In the model outlined above, it is implicitly assumed that the cameras involved are calibrated. An indeed, the chessboard-based camera calibration approach outlined in Subsection 5.2 was used for this purpose. The resulting camera positions and orientations were as expected. However, it soon turned out that the calibration of the multi-camera arrangement - based on these individual camera calibrations only - does not ensure the precision required for the specular surface reconstruction. Therefore, a second - fine-tuning - calibration step proved to be necessary. A joint and specularity-based calibration of the cameras in the topographer arrangement was accomplished. The simulation programs that were developed for modelling reflections of smooth specular surfaces were used for this purpose. A spherical artificial cornea with know radius was used as a secondary fiducial in this case.

Again, the Levenberg-Marquardt method - mentioned earlier in Subsection 5.2 - was used to fine-tune the vector of parameters that included, in this case, all the extrinsic camera parameters of all the three cameras and the position and orientation of the test cornea. The aim was to minimize the total sum of quadratic error between the blob-centres in the simulated reflection images and the detected ones. 

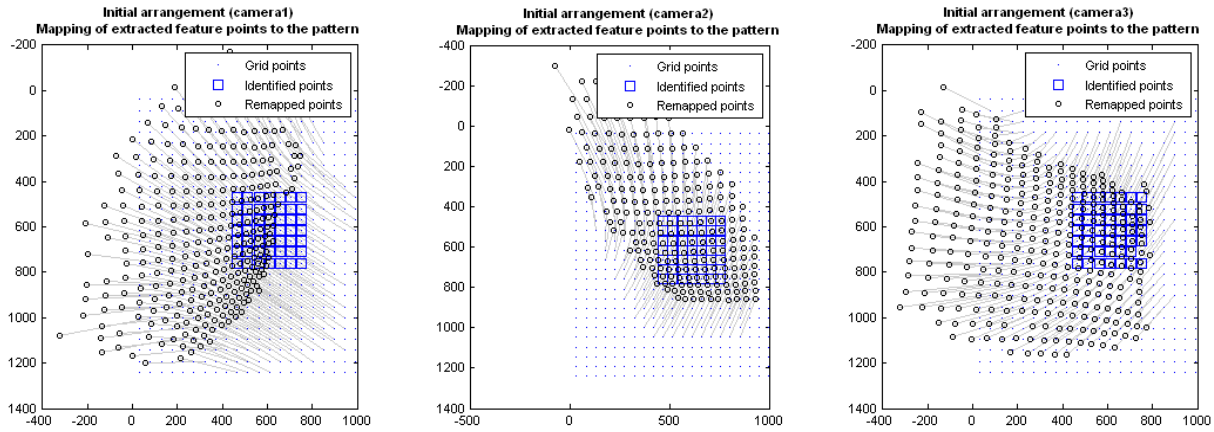

Fig. 14. The importance of the joint camera calibration is indicated by the considerable change in the back-projected grid point locations (i.e., locations derived by back-projecting the detected points onto the measurement pattern's plane). The o's mark the grid-point locations according to the individual camera calibration results (i.e., before the joint camera calibration), while the $\cdot$ 's indicate the grid point locations after the joint camera calibration.

The optimization resulted in the correction of the camera parameters. The need for this finetuning step is underlined by the significant (measurement pattern) point location-changes shown in the final plane of measurement pattern. Such point location-changes are shown for each of the cameras in Figure 14.

\subsection{Surface reconstruction via solving the partial differential equation. Choosing an advantageous parameterization}

A numerical procedure was devised to solve the above PDE's. The solution of these PDE's in effect conveys the reconstructed corneal surface. The solution involves transcribing the PDE's for individual 'rows' and the 'columns. In this manner, first-order ordinary nonlinear differential equations were generated. These were then numerically integrated using the well-known Runge-Kutta method - along 'row' and 'column' directions, as necessary.

Figure 15 illustrates a conceptual processing direction - e.g., from left to right in a particular row of the measurement pattern - and corresponding curves on the specular surface $F$ and in the image plane of one of the cameras, as an ordinary differential equation derived from the PDE is solved. 


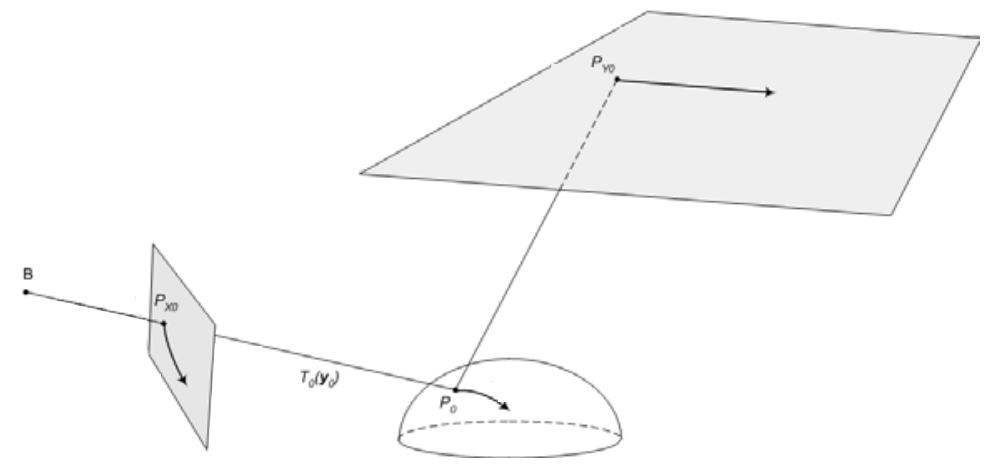

Fig. 15. Directions followed explicitly - in this case on the measurement pattern's plane - or implicitly - on the specular surface and in the image plane - during the numerical integration of the partial differential equation describing the surface reconstruction problem.

Starting conditions are necessary to obtain realistic solution for the PDE given in Subsection 5.1. These were computed with the method outlined in the next subsection. An efficient computational scheme was devised to re-use the points computed earlier to improve reconstruction speed. The reconstruction paths used in this scheme is shown in Figure 19 for one of the cameras.
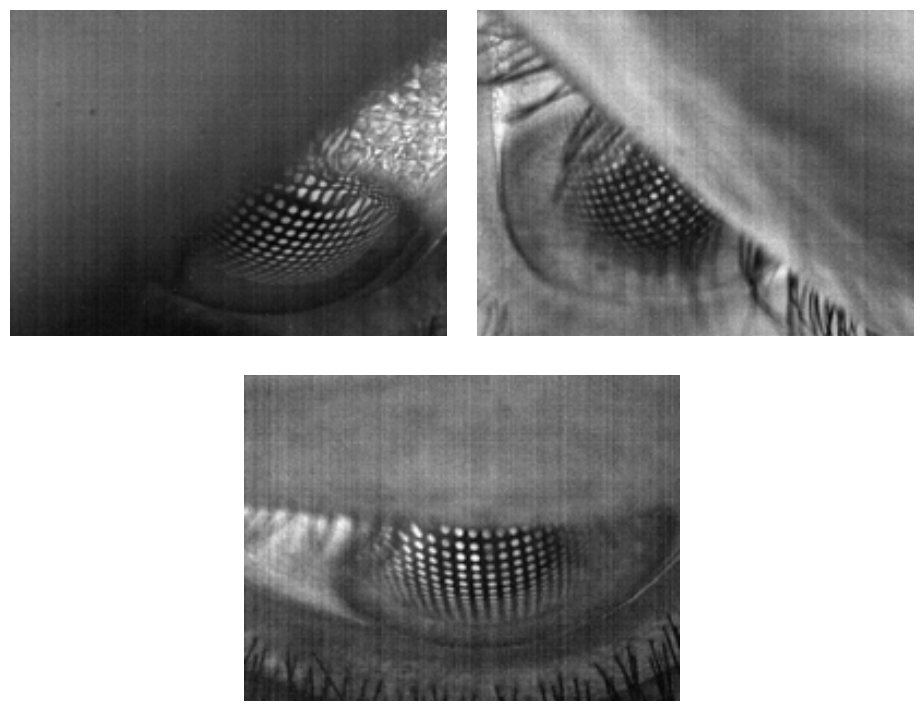

Fig. 16. Purkinje-images of the planar measurement pattern as "seen" by the three cameras of the topographer arrangement. 

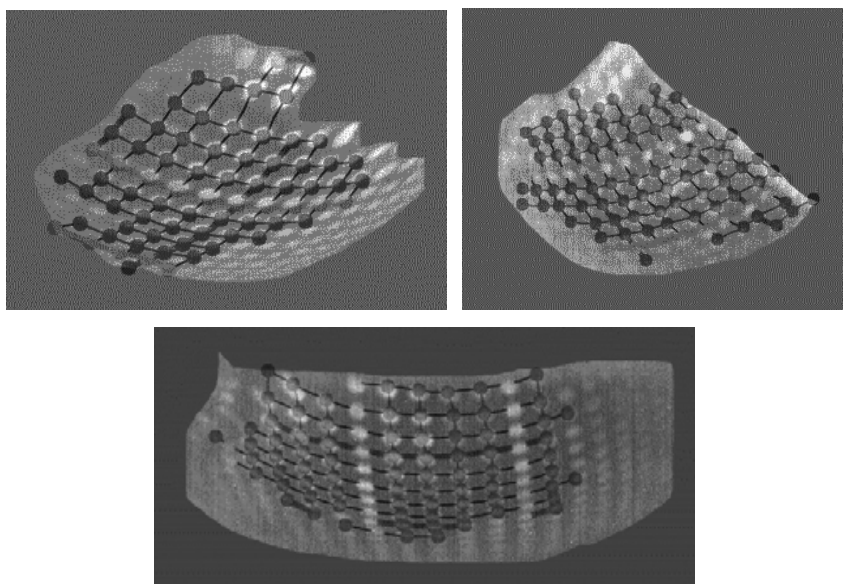

Fig. 17. The grid of the detected blobs overlaid on the corresponding image segments.

\subsection{Setting of starting condition}

The exact value of the above starting condition could be found out by including two laserlights for each camera (as it is frequently done in other topographers). However, this distance setting mechanism is unnecessary here, as precise stereo information can be gathered with two calibrated cameras looking at the same patch of specular surface (a situation illustrated in Figure 13). An assumed surface-point and the unit normal vector at this point determined from the first reflection image - are used to determine the point in the measurement pattern that should be seen in the second image. See Figure 18.

The Euclidean distance between the 'should-be-seen' point and the point really seen by the second camera (i.e., the length of the segment marked red in Figure 18) is calculated. If this distance (error) is considerable for an assumed surface-point, then it is - in reality - off the specular surface $F$.

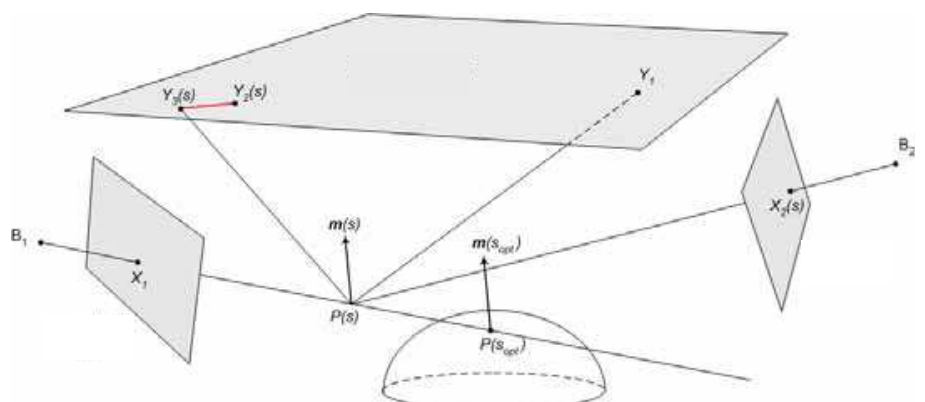

Fig. 18. Locating a point of a specular surface in the space based on two views. 


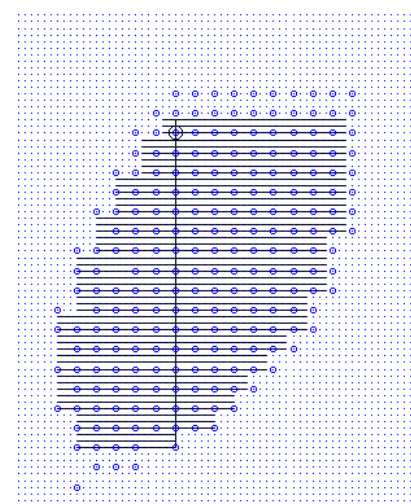

Fig. 19. Surface reconstruction paths - in the plane of the measurement pattern - for the surface region "seen" by one of the three cameras.

By moving along possible surface-points and considering also their unit normal vectors that are for the first camera, the aforementioned distance must be minimized.

The near-zero valued minima mark the spatial positions of surface-points that can be used as stating points for the reconstruction.

\subsection{Reconstruction results}

In Figure 21, the reconstruction precision achieved in case of a test cornea is shown. Clearly, the joint and specularity-based calibration resulted in usable positions for the cameras. In Figure 20, the distances of the reconstructed surface points from the spherical surface of the test cornea is plotted. The mentioned error distance values in peripheral areas - far from the starting point of the processing - seem to be relatively high (in the order of $10 \mu \mathrm{m}$ ).

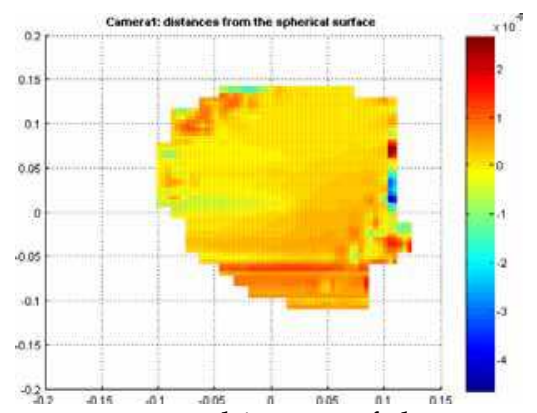

Fig. 20. Reconstruction errors encountered in case of the test cornea shown before for the surface region "seen" by one of the cameras. 

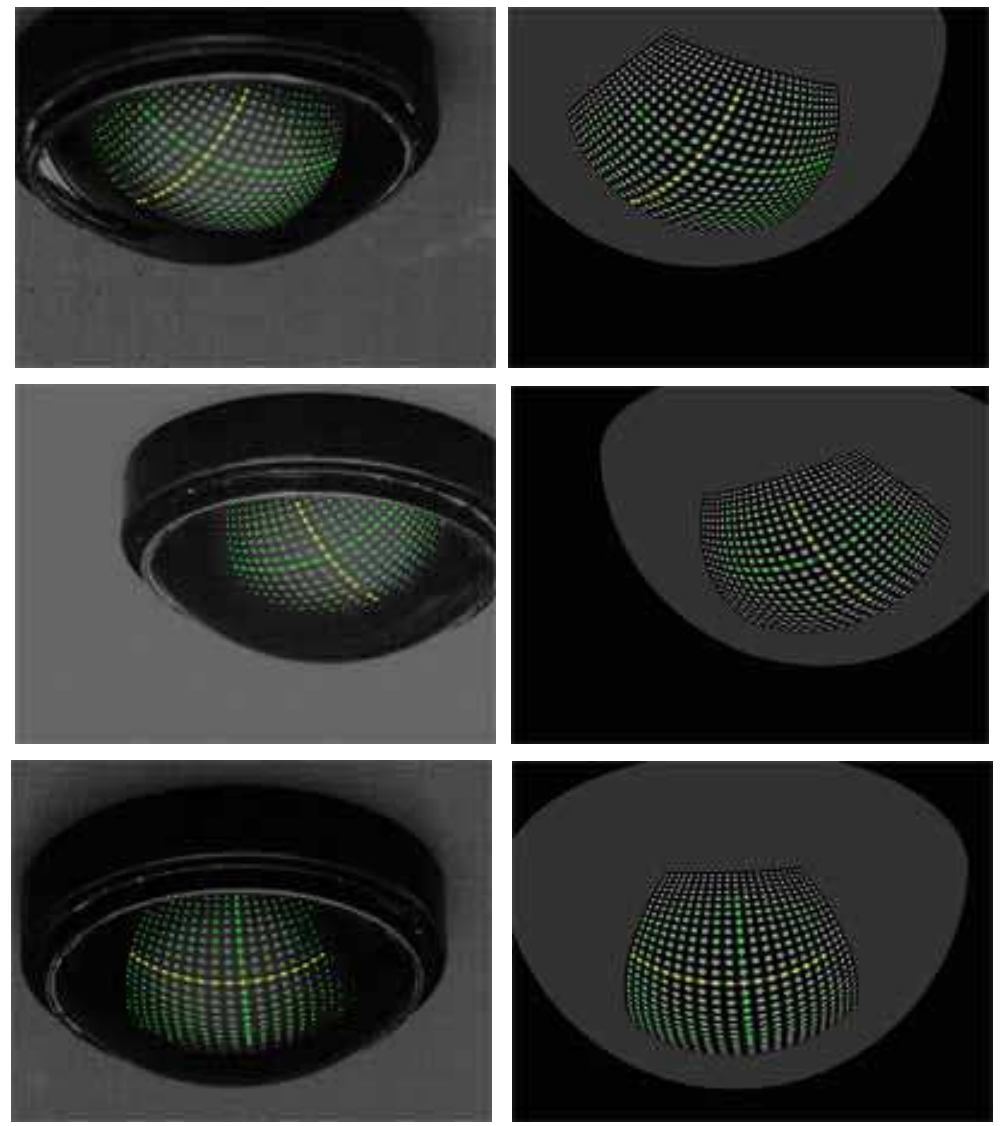

Fig. 21. Virtual images - formed by a test cornea - taken with the three cameras of the topographer arrangement (left). The detected blobs are marked with their centroids. Corresponding syntetic images (right) created via ray-tracing.

\section{Conclusions and further work}

The majority of the topographers in use today, rely on one view only, which is theoretically insufficient for the unique reconstruction of the corneal surface. To overcome this essential measurement deficiency, a multi-camera arrangement was proposed by the authors and mathematical methods were devised for the specular surface reconstruction. However, further experiments and simulations are required to improve the surface coverage of the multi-camera arrangement.

\section{Acknowledgements}

This research has been partially supported by the National Office for Research and Technoogy (NORT), Hungary, in the frame of the NKFP-2/020/04 research contract. 


\section{References}

ANSI (2008). Corneal Topography Systems - Standard Terminology, Requirements, Z80.23-2008, American National Standards Institute, Washington, DC, USA

Bódis-Szomorú, A.; Dabóczi, T. \& Fazekas, Z. (2008). Calibration and sensitivity analysis of a stereo vision-based driver assistance system, In: Stereo Vision, Bhatti, A. (Ed.), pp. 126, InTech Education and Publishing, ISBN 978-953-7619-22-0, Vienna, Austria

Bonfort, T. \& Sturm, P. (2003). Voxel carving for specular surfaces, Proceedings of the 9th IEEE International Conference on Computer Vision, pp. 691-696, ISBN 0-7695-1950-4, Nice, France, October 2003, IEEE Computer Society, Washington, DC

Corbett, M. C.; Rosen, E. S.; \& O'Brart, D. P. S. (1999). Corneal Topography: Principles and Applications, BMJ Publishing, ISBN 0-7279-1226-7, London, UK

de Almeida, M. S. \& Carvalho, L. A. (2007). Different schematic eyes and their accuracy to the in vivo eye: a quantitative comparison study. Brazilian Journal of Physics, Vol. 37, No. 2A, pp. 378-387, ISSN 0103-9733

Fazekas, Z.; Soumelidis, A.; Bódis-Szomorú, A. \& Schipp, F. (2008). Specular surface reconstruction for multi-camera corneal topographer arrangements, Proceedings of the 30th Annual International IEEE EMBS Conference, pp. 2254-2257, ISBN 978-1-42441814-5, Vancouver, British Columbia, Canada, August 2008, IEEE EMBS, Piscataway, NJ, USA

Fleming, R. W.; Torralba, A. \& Adelson, E. H. (2004). Specular reflections and the perception of shape. Journal of Vision, Vol. 4, No. 9, pp. 798-820, ISSN 1534-7362

Griffin, P. M.; Narasimhan, S. \& Yee, S. R. (1992). Generation of uniquely encoded light patterns for range data acquisition. Pattern Recognition, Vol. 25, No. 6, pp. 609-616, ISSN 0031-3203

Halstead, M. A.; Barsky, B. A.; Klein, S. A. \& Mandell, R. B. (1996). Reconstructing curved surfaces from specular reflection patterns using spline surface fitting to normals, Proceedings of the 23rd Annual Conference on Computer Graphics and Interactive Techniques, pp. 335-342, ISBN 0-89791-746-4, New Orleans, Louisiana, USA, August 1996, ACM, New York

Hartley, R. \& Zissermann, A. (2004). Multiple View Geometry in Computer Vision, (2nd edition) Cambridge University Press, ISBN 0-5215-4051-8, Cambridge, UK

Iskander, D.R.; Collins, M. J. \& Davis, B. (2000). Optimal modeling of corneal surfaces with Zernike polynomials. IEEE Transactions on Biomedical Engineering, Vol. 48, No. 1, pp. 87-95, ISSN 0018-9294

Jongsma, F.; de Brabander, J. \& Hendrikse, F. (1999). Review and classification of corneal topographers. Lasers in Medical Science, Vol. 14, No. 1, pp. 2-19, ISSN 0268-8921

Kickingereder, R. \& Donner, K, (2004). Stereo vision on specular surfaces, Proceedings the 4th IASTED International Conference on Visualization, Imaging, and Image Processing, pp. 335-339, ISBN 0-88986-454-3, Marbella, Spain, September 2004, IASTED, Calgary, Alberta, Canada

Lellmann, J.; Balzer, J.; Rieder, A. \& Beyerer, J. (2008). Shape from Specular Reflection and Optical Flow. International Journal of Computer Vision, Vol. 80, No. 2, pp. 226-241, ISSN 0920-5691

Németh, J.; Erdélyi, B.; Csákány, B.; Gáspár, P.; Soumelidis, A.; Kahlesz, F. \& Lang, Zs. (2002). High-speed videotopographic measurement of tear film build-up time. Investigative Ophthalmology and Visual Science, Vol. 43, pp. 1783-1790, ISSN 0146-0404 
Savarese, S. \& Perona, P. (2001). Local analysis for 3D reconstruction of specular surfaces, Proceedings of IEEE Computer Society Conference on Computer Vision and Pattern Recognition, Vol. 2, pp. 738-745, ISBN 0-7695-1272-0, Kauai, Hawaii, USA, December 2001, IEEE Computer Society, Washington, DC

Savarese, S. \& Perona, P. (2002a). Local analysis for 3D reconstruction of specular surfaces Part II, Proceedings of the 7th European Conference on Computer Vision, Lecture Notes in Computer Science, Vol. 2351, pp. 1148-1158, ISBN 3-540-43744-4, Copenhagen, Denmark, May 2002, Springer, Heidelberg

Savarese, S.; Chen, M. \& Perona, P. (2002b). Second order local analysis for 3D reconstruction of specular surfaces, Proceedings of the First International Symposium on 3D Data Processing Visualisation and Transmission, pp. 356-361, ISBN 0-7695-1521-4, Padova, Italy, June 2002, IEEE Computer Society, Washington, DC

Savarese, S.; Chen, M. \& Perona, P. (2004). What do reflections tell us about the shape of a mirror? Proceedings of the 1st Symposium on Applied Perception in Graphics and Visualization, pp. 115-118, ISBN 1-58113-914-4, Los Angeles, California, USA, August 2004, ACM, New York

Sicam, V. A; Snellenburg, J. J.; van der Heide, R. G. L. \& van Stokkum, I. H. M. (2007). Pseudo Forward Ray-Tracing: A new method for surface validation in cornea topography. Optometry \& Vision Science, Vol. 84, No. 9, pp. E915-E923, ISSN 1040-5488

Soumelidis, A.; Fazekas, Z.; Schipp, F.; Edelmayer, A.; Németh, J. \& Csákány, B. (2008). Development of a multi-camera corneal topographer using an embedded computing approach, Proceedings of the 1st International Conference on Biomedical Electronics and Devices, pp. 126-129, ISBN 978-989-8111-17-3, Funchal, Madeira, Portugal, Jan. 2008, INSTICC, Setúbal, Portugal

Swartz, T.; Marten, L. \& Wang, M. (2007). Measuring the cornea: the latest developments in corneal topography. Current Opinion in Ophthalmology., Vol. 18, No. 4, pp. 325-333, ISSN 1040-8738

Trichet, R.. \& Merialdo, B. (2007). Probabilistic matching algorithm for keypoint based object tracking using a Delaunay triangulation, Proceedings of the Eight International Workshop on Image Analysis for Multimedia Interactive Services, pp. 17-20, ISBN 0-76952818-X, Santorini, Greece, June 2007, IEEE Computer Society, Washington, DC, USA

Vos, F. M.; van der Heijde, G.L.; Spoelder, H.J.W.; van Stokkum, I.H.M. \& Groen, F.C.A. (1997). A new instrument to measure the shape of the cornea based on pseudorandom color coding. IEEE Transactions on Instrumentation and Measurement, Vol. 46, No. 4, pp. 794-797, ISSN 0018-9456

Wang, W.; Wang, Z.; Wang, Y. \& Zuo, T. (2006). Optical aberrations of the cornea and the crystalline lens. Optik - International Journal for Light and Electron Optics, Vol. 117, No. 9, pp. 399-404, ISSN 0030-4026

Zhang, Z. (2000). A flexible new technique for camera calibration. IEEE Transactions on Pattern Analysis and Machine Intelligence, Vol. 22, No. 11, pp. 1330-1334, ISSN 0018-9340 


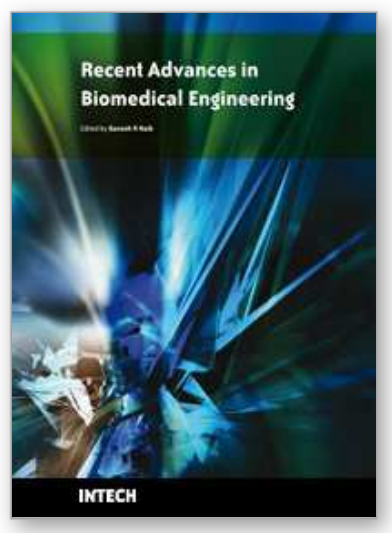

\author{
Recent Advances in Biomedical Engineering \\ Edited by Ganesh R Naik
}

ISBN 978-953-307-004-9

Hard cover, 660 pages

Publisher InTech

Published online 01, October, 2009

Published in print edition October, 2009

The field of biomedical engineering has expanded markedly in the past ten years. This growth is supported by advances in biological science, which have created new opportunities for development of tools for diagnosis and therapy for human disease. The discipline focuses both on development of new biomaterials, analytical methodologies and on the application of concepts drawn from engineering, computing, mathematics, chemical and physical sciences to advance biomedical knowledge while improving the effectiveness and delivery of clinical medicine. Biomedical engineering now encompasses a range of fields of specialization including bioinstrumentation, bioimaging, biomechanics, biomaterials, and biomolecular engineering. Biomedical engineering covers recent advances in the growing field of biomedical technology, instrumentation, and administration. Contributions focus on theoretical and practical problems associated with the development of medical technology; the introduction of new engineering methods into public health; hospitals and patient care; the improvement of diagnosis and therapy; and biomedical information storage and retrieval. The book is directed at engineering students in their final year of undergraduate studies or in their graduate studies. Most undergraduate students majoring in biomedical engineering are faced with a decision, early in their program of study, regarding the field in which they would like to specialize. Each chosen specialty has a specific set of course requirements and is supplemented by wise selection of elective and supporting coursework. Also, many young students of biomedical engineering use independent research projects as a source of inspiration and preparation but have difficulty identifying research areas that are right for them. Therefore, a second goal of this book is to link knowledge of basic science and engineering to fields of specialization and current research. The editor would like to thank the authors, who have committed so much effort to the publication of this work.

\title{
How to reference
}

In order to correctly reference this scholarly work, feel free to copy and paste the following:

A. Soumelidis, Z. Fazekas, A. Bodis-Szomoru, F. Schipp, B. Csakany and J. Nemeth (2009). Specular Surface Reconstruction Method for Multi-Camera Corneal Topographer Arrangements, Recent Advances in Biomedical Engineering, Ganesh R Naik (Ed.), ISBN: 978-953-307-004-9, InTech, Available from: http://www.intechopen.com/books/recent-advances-in-biomedical-engineering/specular-surfacereconstruction-method-for-multi-camera-corneal-topographer-arrangements

\section{INTECH}

open science | open minds

\section{InTech Europe}

University Campus STeP Ri

\section{InTech China}

Unit 405, Office Block, Hotel Equatorial Shanghai 
Slavka Krautzeka 83/A

51000 Rijeka, Croatia

Phone: +385 (51) 770447

Fax: +385 (51) 686166

www.intechopen.com
No.65, Yan An Road (West), Shanghai, 200040, China 中国上海市延安西路65号上海国际贵都大饭店办公楼405单元 Phone: +86-21-62489820

Fax: +86-21-62489821 
(C) 2009 The Author(s). Licensee IntechOpen. This chapter is distributed under the terms of the Creative Commons Attribution-NonCommercial-ShareAlike-3.0 License, which permits use, distribution and reproduction for non-commercial purposes, provided the original is properly cited and derivative works building on this content are distributed under the same license. 